\title{
The Detector System for the Stratospheric Kinetic Inductance Polarimeter (SKIP)
}

\author{
B. R. Johnson ${ }^{1}$, P. A. R. Ade ${ }^{4}$, D. Araujo ${ }^{1}$, K. J. Bradford ${ }^{1}$, D. Chapman ${ }^{1}$, \\ P. K. Day ${ }^{7}$, J. Didier ${ }^{1}$, S. Doyle ${ }^{4}$, H. K. Eriksen ${ }^{5}$, D. Flanigan ${ }^{1}$, C. Groppi ${ }^{2}$, \\ S. Hillbrand ${ }^{1}$, G. Jones ${ }^{6,1}$, M. Limon ${ }^{1}$, P. Mauskopf ${ }^{2}$, H. McCarrick ${ }^{1}$, \\ A. Miller ${ }^{1}$, T. Mroczkowski ${ }^{3}$, B. Reichborn-Kjennerud ${ }^{1}$, B. Smiley ${ }^{1}$, \\ J. Sobrin ${ }^{1}$, I. K. Wehus ${ }^{7}$, J. Zmuidzinas ${ }^{3,7}$ \\ 1) Department of Physics, Columbia University, New York, NY 10027, USA \\ 2) Department of Physics, Arizona State University, Tempe, AZ 85287, USA \\ 3) Department of Physics, Caltech, Pasadena, CA 91125, USA \\ 4) School of Physics and Astronomy, Cardiff University, Cardiff, Wales, CF243YB, UK \\ 5) Institute of Theoretical Astrophysics, University of Oslo, N-0315 Oslo, Norway \\ 6) National Radio Astronomy Observatory, Charlottesville, VA 22903, USA \\ 7) Jet Propulsion Laboratory, Caltech, Pasadena, CA 91109, USA
}

July 31st, 2013

\begin{abstract}
The Stratospheric Kinetic Inductance Polarimeter (SKIP) is a proposed balloon-borne experiment designed to study the cosmic microwave background, the cosmic infrared background and Galactic dust emission by observing 1133 square degrees of sky in the Northern Hemisphere with launches from Kiruna, Sweden. The instrument contains 2317 single-polarization, horn-coupled, aluminum lumpedelement kinetic inductance detectors (LEKIDS). The LEKIDS will be maintained at $100 \mathrm{mK}$ with an adiabatic demagnetization refrigerator. The polarimeter operates in two configurations, one sensitive to a spectral band centered on $150 \mathrm{GHz}$ and the other sensitive to 260 and $350 \mathrm{GHz}$ bands. The detector readout system is based on the ROACH-1 board, and the detectors will be biased below $300 \mathrm{MHz}$. The detector array is fed by an F/2.4 crossed-Dragone telescope with a $500 \mathrm{~mm}$ aperture yielding a 15 arcmin FWHM beam at $150 \mathrm{GHz}$. To minimize detector loading and maximize sensitivity, the entire optical system will be cooled to $1 \mathrm{~K}$. Linearly polarized sky signals will be modulated with a metal-mesh half-wave plate that is mounted at the telescope aperture and rotated by a superconducting magnetic bearing. The observation program consists of at least two, five-day flights beginning with the $150 \mathrm{GHz}$ observations.
\end{abstract}

\section{Introduction}

The cosmic microwave background radiation (CMB) carries an image of the universe as it was 380,000 years after the Big Bang. Measurements of the angular intensity and polarization anisotropies in the CMB have proven to be a treasure trove of cosmological information. For example, CMB measurements have helped reveal that spacetime is flat, the universe is 13.8 billion years old, and it is dominated by cold dark matter and dark energy. A faint and yet-to-be-detected divergence-free "B-mode" polarization anisotropy signal in 


\begin{tabular}{|l|c|c|}
\hline Instrument Configuration & Flight 1 & Flight 2 \\
\hline Spectral Band Centers $[\mathrm{GHz}]$ & 150 & 267,350 \\
Spectral Bandwidth $[\delta v / v]$ & 0.27 & $0.22,0.30$ \\
Number of Detectors & 2317 & 1655,662 \\
Total Number of Detectors & 2317 & 2317 \\
Detector NET $[\mu \mathrm{K} \sqrt{\mathrm{sec}}]$ & 58.4 & 149,354 \\
Instrument NET $[\mu \mathrm{K} \sqrt{\mathrm{sec}}]$ & 1.24 & $3.76,14.1$ \\
Aperture Diameter $[\mathrm{mm}]$ & 500 & 280 \\
F/\# & 2.4 & 4.3 \\
Beam FWHM [arcmin] & 14.5 & $14.5,11.3$ \\
Total Sky Coverage $\left[\mathrm{deg}^{2}\right]$ & 1133 & 1133 \\
$\ell$ Range & 10 to 1000 & 10 to 1000 \\
Flight Duration [days] & 5 & 5 \\
Time on CMB Patch $[$ hours] & 102.7 & 102.7 \\
Duty Cycle [\%] & 86 & 86 \\
T sensitivity per $1^{\circ}$ pixel $[\mu \mathrm{K}]$ & 0.069 & $0.21,0.78$ \\
Q sensitivity per $1^{\circ}$ pixel $[\mu \mathrm{K}]$ & 0.097 & $0.29,1.1$ \\
\hline Minimum $r(99 \%$ confidence $)$ & \multicolumn{2}{|c|}{0.02} \\
\hline
\end{tabular}

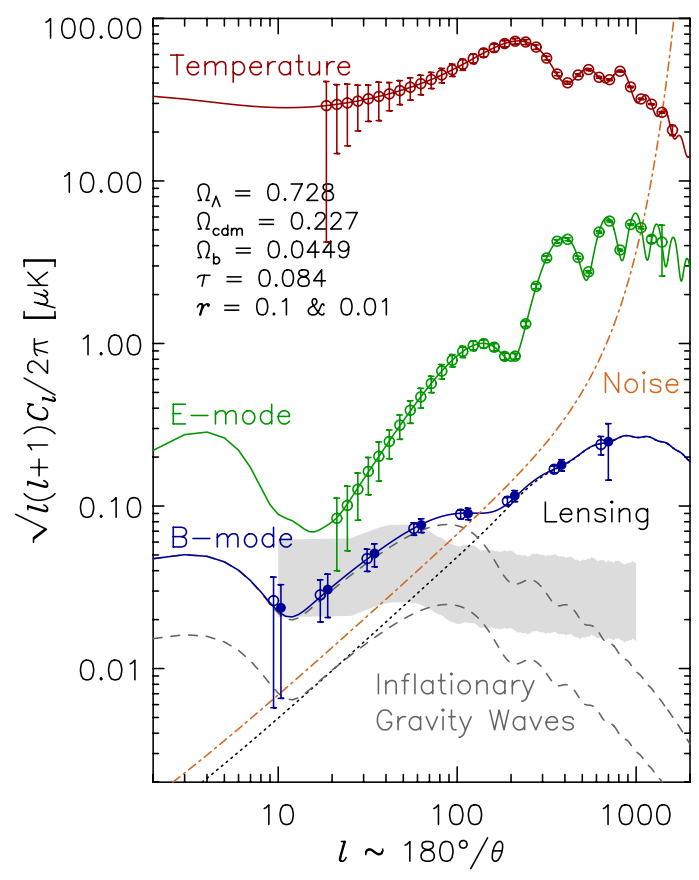

Figure 1: Left: Key SKIP performance characteristics. Right: Theoretical angular power spectra for the CMB signals and the expected SKIP performance. For the B-modes, the open circles show the raw sensitivity of the $150 \mathrm{GHz}$ flight alone ignoring foreground component separation, while the closed circles include sensitivity degradation due to component separation. The grey region shows the spectrum of foreground signals from Galactic dust assuming the polarization fraction is between $5 \%$ and $15 \%$ as predicted[4].

the CMB should contain additional information; B-modes generated when CMB photons are gravitationally lensed by large-scale structure will yield the values of physical parameters such as the sum of the neutrino masses, and if discovered, an inflationary gravity-wave (IGW) B-mode signal would reveal the energy scale at which inflation occurred[1]. SKIP is a proposed balloon-borne experiment designed with the sky coverage, angular resolution, sensitivity and spectral coverage needed to either measure or constrain the amplitude of the IGW signal to approximately the foreground confusion limit set by the aforementioned gravitational lensing signal. The table in Figure 1 shows the experiment characteristics and the plot shows the forecasted CMB power spectra results that will come from SKIP.

To isolate the faint B-mode signals, brighter foreground signals, such as Galactic dust emission, must be precisely measured and removed from the CMB maps. To disentangle the various sky signals, SKIP will observe a low-dust region in the Northern Hemisphere with three spectral bands, and then fit a frequencydependent parametric dust model to the data pixel-by-pixel using standard likelihood techniques[2]. The target sky patch is accessible with launches from Kiruna, Sweden.

Because the Galactic dust emission is so faint in the SKIP patch, the $350 \mathrm{GHz}$ spectral band will also be able to image angular fluctuations in the cosmic infrared background (CIB)[3]. The CIB was emitted by dust grains in distant galaxies, and it has a redshifted, dust-like frequency spectrum. The CIB probes the matter distribution on redshifts $z \leq 6$, rather than at $z \sim 1100$ like the CMB, so it can be used to constrain star formation history and dark matter distribution.

Finally, SKIP will investigate whether lumped-element kinetic inductance detectors (LEKIDS) are a candidate detector technology for a future CMB satellite mission. The SKIP instrument is described in Section 2 , the detector system is described in Section 3, and the observation program is described in Section 4 . 


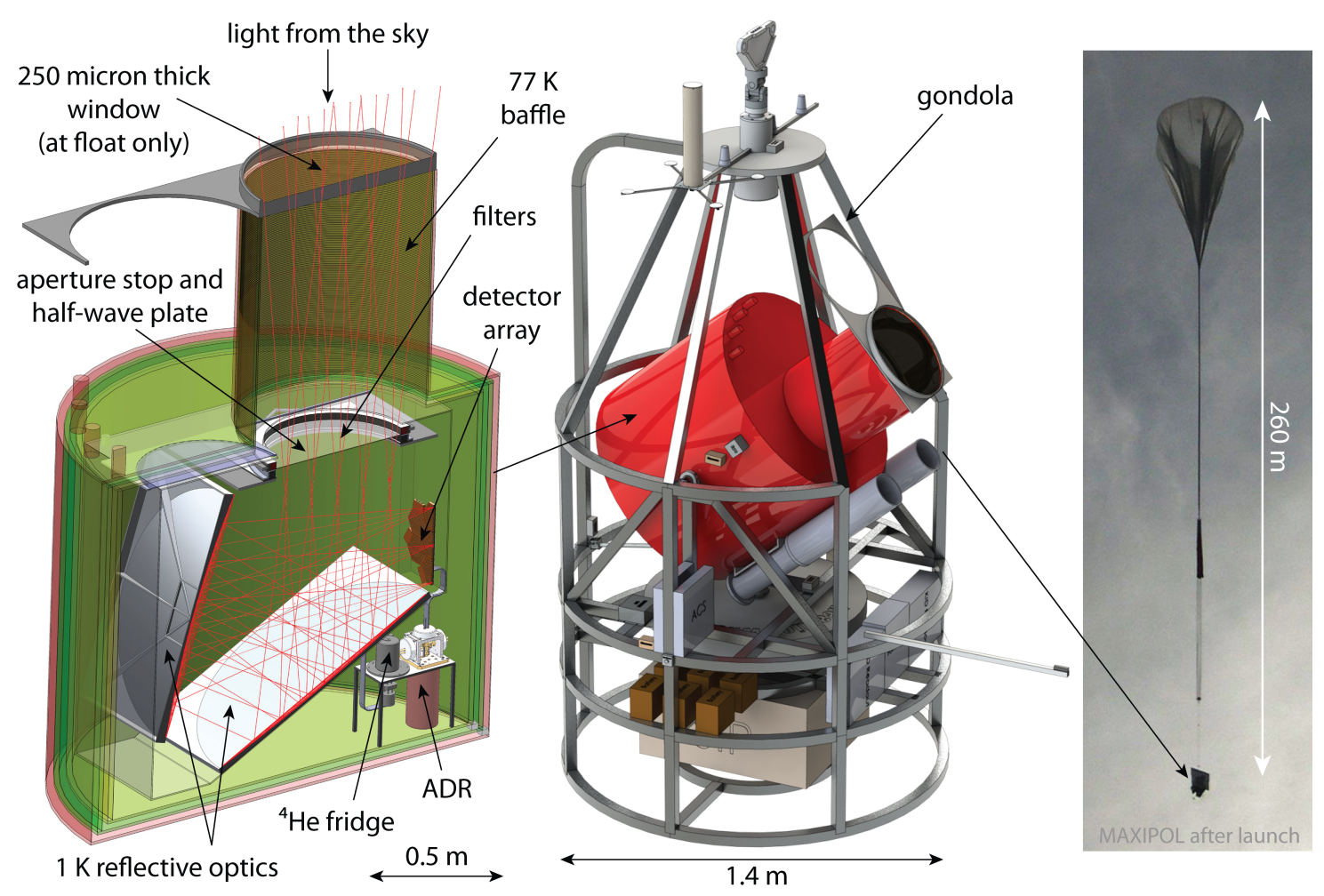

Figure 2: The balloon-borne SKIP instrument. Left: A cross-sectional view of the polarimetric receiver. Center: The receiver mounted in the gondola. Right: An example photograph of a balloon-borne instrument after launch.

\section{Instrument Details}

The SKIP polarimeter is based on a catoptric crossed-Dragone telescope composed of an off-axis parabolic primary mirror and a hyperbolic secondary mirror yielding an effective focal length of $1200 \mathrm{~mm}$ (see Figure 2). A $500 \mathrm{~mm}$ diameter aperture stop, that is cooled to $1 \mathrm{~K}$, produces a 15 arcmin FWHM beam at $150 \mathrm{GHz}$. A metal-mesh half-wave plate (HWP) mounted at this aperture stop will rotate at $7.4 \mathrm{~Hz}$ using a drive system based on a superconducting magnetic bearing (SMB), which minimizes vibrations and eliminates heating from stick-slip friction. When paired with the downstream analyzer, which is part of the detector array, the linearly polarized component of the sky signals will be modulated at four times the rotation frequency of the HWP in the data stream $(29.7 \mathrm{~Hz})$. The detector array, which contains 2317 single-polarization, horn-coupled, aluminum LEKIDS, is mounted at the focal plane of the telescope.

To minimize detector loading and maximize sensitivity, the entire polarimeter will be mounted inside a cryostat with liquid nitrogen and liquid helium stages. A box enclosing the optics will be thermally isolated from the liquid helium stage of this cryostat by Vespel legs and maintained at $1 \mathrm{~K}$ using a Chase Cryogenics closed-cycle ${ }^{4} \mathrm{He}$ refrigerator, which is capable of providing $500 \mu \mathrm{W}$ of cooling power for five days. The detector array is mounted inside this $1 \mathrm{~K}$ optics box using thermally insulating Vespel legs, and it is cooled to $100 \mathrm{mK}$ by a two-stage adiabatic demagnetization refrigerator (ADR). The ADR contains a ferric ammonium alum (FAA) paramagnetic salt pill with a $0.12 \mathrm{~J}$ capacity when regulated at $100 \mathrm{mK}$, backed by a gallium gadolinium garnet (GGG) salt pill with a $1.2 \mathrm{~J}$ capacity capable of cooling to $800 \mathrm{mK}$. Optical loading is controlled by (i) a radiation shield in the "snout" of the receiver held at $77 \mathrm{~K}$, which is designed to control far side-lobes, (ii) a second radiation shield held at the liquid helium bath temperature $(\sim 1.5 \mathrm{~K}$, see below) designed to terminate power from the horns down to $-20 \mathrm{~dB}$, (iii) a series of low-pass metal-mesh 

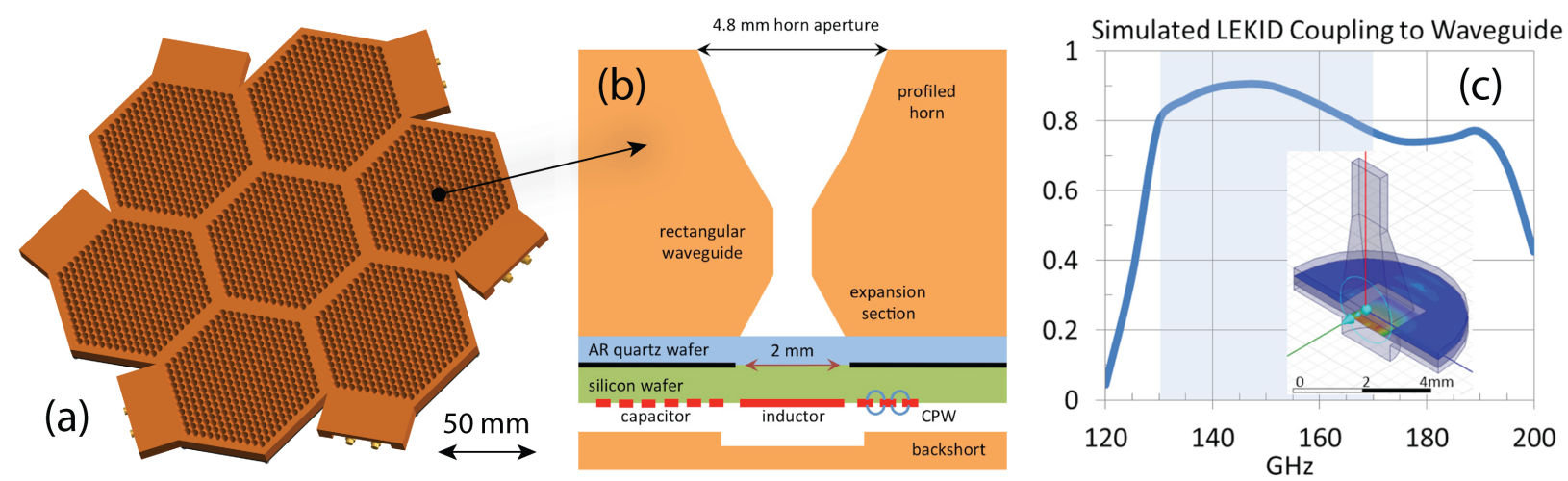

Figure 3: The SKIP detector array. Left: The focal plane is composed of seven modules each containing 331 horn-coupled detectors. Center: A cross-sectional view of one focal plane pixel shows the profiled horn, the single-moded waveguide and the LEKID. Right:. A plot showing the detector absorptance as simulated by the Ansoft Software package HFSS.

filters that reject high-frequency radiative power from the sky, and (iv) the receiver uses a double window mechanism based on designs that have flown successfully in the past [5, 6] in which a retractable thick window supports the differential pressure between the inside and outside of the cryostat on the ground, while a thin $(250 \mu \mathrm{m})$ window is revealed at float. The polarimeter operates in two configurations, one sensitive to a single spectral band centered on $150 \mathrm{GHz}$ and the other sensitive to 260 and $350 \mathrm{GHz}$ bands. The only differences between configurations are (i) the band-defining filters, (ii) the single-moded waveguide section of the horn, (iii) the aperture stop diameter, and (iv) the half-wave plate.

The polarimeter is housed in a robust gondola designed for multiple flights. The balloon and the instrument will reach an altitude of 100-125 kft, where the ambient pressure is 5-13 mbar. We regulate the nitrogen vapor to atmospheric pressure and allow the helium bath to reach ambient pressure at float, resulting in a nitrogen stage temperature of $77 \mathrm{~K}$ and a helium stage temperature of approximately $1.5 \mathrm{~K}$.

\section{LEKID Detector System}

The SKIP focal plane architecture is shown in Figure 3. The focal plane diameter is $\sim 300 \mathrm{~mm}$, and it consists of seven hexagonal modules. Each module contains 331 horn-coupled LEKIDS, for a total of 2317 detectors. All of the detectors in a single module are frequency-multiplexed in a 135-270 MHz readout band, allowing the entire focal plane to be read out using only seven SiGe bipolar cryogenic low noise amplifiers (LNAs) [7] and seven pairs of coaxial cables.

We use horns to couple sky signals to the detectors. As shown in Figure 3b, the horn narrows down to a single-mode rectangular waveguide section, which reduces spillover and stray-light loading inside the optics box and provides high-performance polarization selection and an integrated high-pass filter. The $1 F \lambda=4.8 \mathrm{~mm}$ horn aperture allows a dense focal plane layout and yields high receiver sensitivity and fast mapping speeds. With this relatively small horn aperture, the instrument beam is primarily defined by the cold aperture stop. The horns couple with approximately $38 \%$ efficiency to the sky at $150 \mathrm{GHz}$, and the remaining $62 \%$ of the horn beam is terminated on the $1 \mathrm{~K}$ optics box which results in negligible loading. The final section of the waveguide is re-expanded to flatten the propagation impedance at the low-frequency edge of the band, which improves optical coupling and allows the radiation to be launched efficiently into the silicon LEKID wafer.

The LEKIDS will be fabricated from $20 \mathrm{~nm}$ thick aluminum films deposited on $300 \mu \mathrm{m}$ thick, high-resistivity silicon substrates. The baseline pixel design consists of a back-illuminated single-polarization LEKID where 

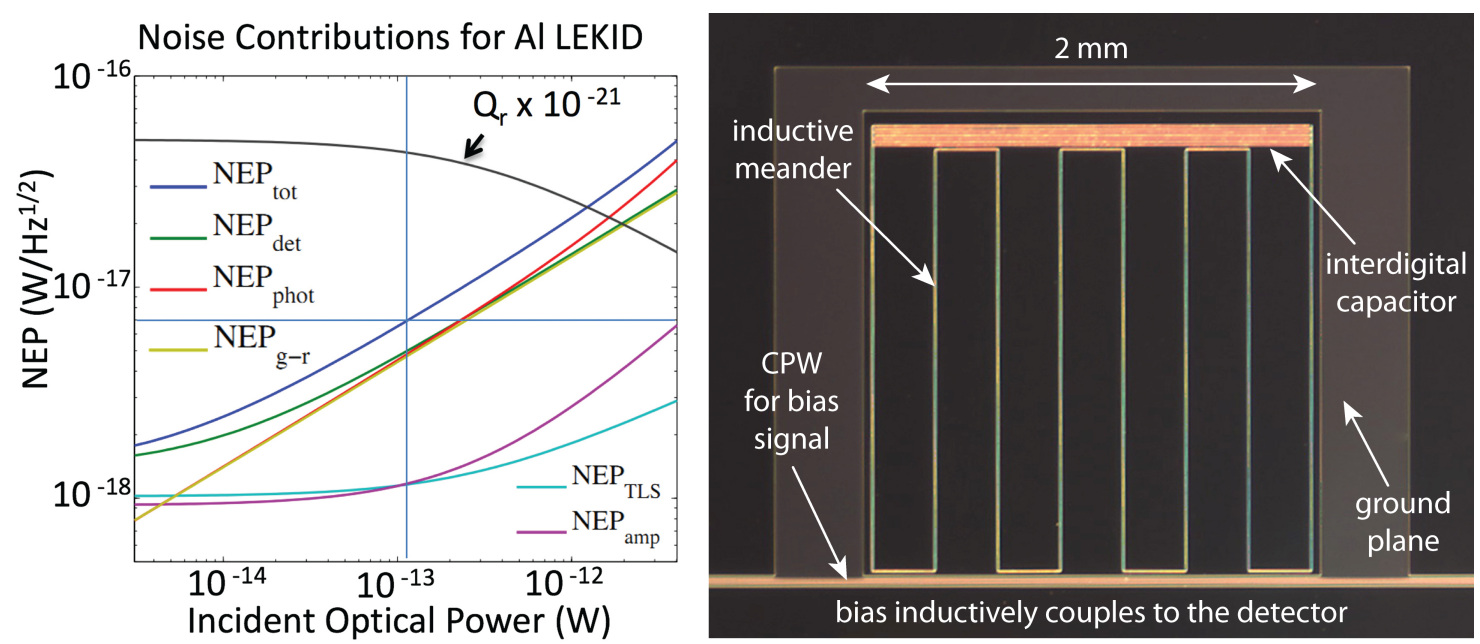

Figure 4: Left: Expected NEP and resonator $Q$ versus incident optical loading. These curves were computed using both theory [10] and measurements[11]. The total NEP $\left(\mathrm{NEP}_{\text {tot }}\right)$ curve includes contributions from the photon loading $\left(\mathrm{NEP}_{\text {phot }}\right)$ and the expected detector noise $\left(\mathrm{NEP}_{\text {det }}\right)$, which includes generation-recombination (g-r) noise, two-level system (TLS) noise, and readout amplifier (amp) noise. The light blue vertical and horizontal rules indicate the expected loading and NEP for SKIP. The resonator $Q$ curve has been multiplied by a factor $10^{-21}$ for display purposes. Right: A photomicrograph of one LEKID.

the inductor/absorber is a meandered aluminum trace on a silicon substrate with a filling factor designed to match the wave impedance (see Figure 4). This design is similar to the absorbers used in the NIKA[8] and MAKO[9] instruments. An anti-reflection coating (ARC) of fused quartz is used to impedance match the silicon wafer and the horn. The ARC and detector wafers are mounted directly to the back of the horn plate and a metal back plate closes each module. The back plate has metal cavities behind each detector that are designed to act as backshorts close to $\lambda / 4$ in length. The small fraction of the radiation that propagates laterally in the two dielectric substrates will be absorbed by a titanium nitride (TiN) film deposited and patterned on the ARC. This TiN layer has apertures in it to allow radiation from the sky to propagate from the horns through the quartz and silicon to the detectors.

The detector readout electronics are based on (i) the ROACH signal processing board, developed by the CASPER collaboration[13], which hosts a Xilinx field-programmable gate array (FPGA) and (ii) the successful Open-Source Readout system developed at Caltech for the MUSIC instrument[12]. The stimulus tones for the LEKID resonators are directly synthesized in the 135-270 MHz band using a continuous playback ring-buffer which drives a 16-bit digital-to-analog converter (DAC). The output of the DAC is amplified and bandpass-filtered to suppress harmonics before being fed to the detector wafer. Digitally controlled attenuators are used to optimally adjust the overall signal levels. After exiting the cryostat, the modulated carrier tones are then further amplified and filtered before being sampled by a 12-bit analog-to-digital converter (ADC). The digitized data is then channelized by the same FPGA. The voltage data from 331 channels which contain resonators will then be sent via Ethernet to data acquisition computers for storage. Figure 5 shows an overview of the SKIP data acquisition electronics.

\section{Observation Plans}

One of the lowest foreground regions at $150 \mathrm{GHz}$ anywhere on the sky is located at Galactic coordinates (1, b) $=\left(99^{\circ}, 75^{\circ}\right)$, which is in the Northern sky[4]. To observe this region, SKIP will be launched from the ESRANGE balloon facility in Kiruna, Sweden $\left(67.9^{\circ} \mathrm{N}, 20.2^{\circ} \mathrm{E}\right)$. A balloon flight from Kiruna allows five days of integration time, and this low-foreground target field can be continuously observed for the entire 

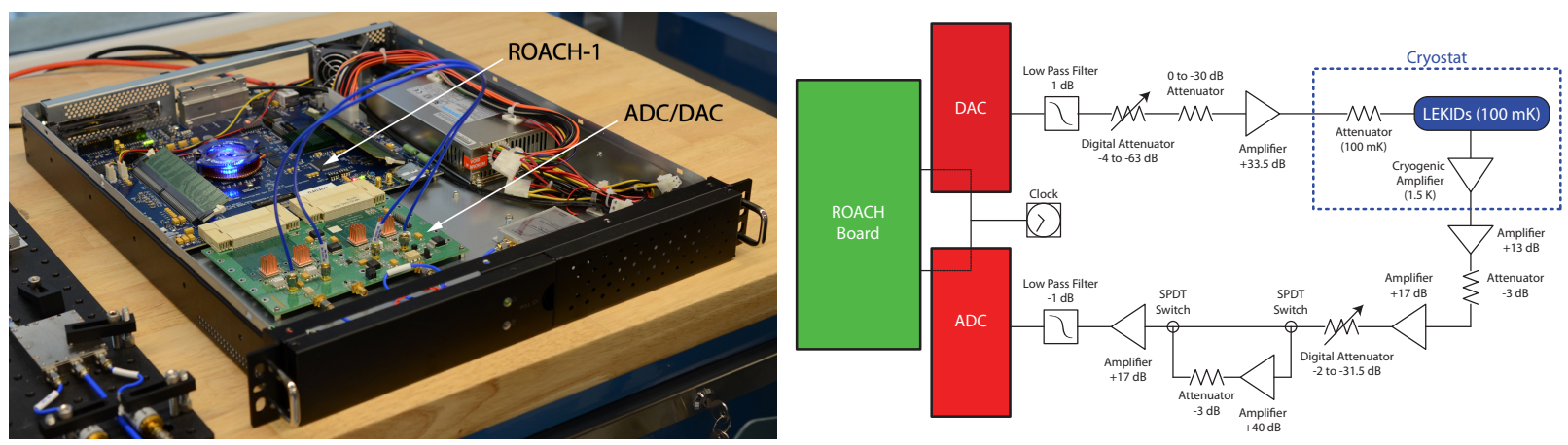

Figure 5: Left: A photograph of a ROACH-I readout currently running at Columbia. Right: A schematic of the SKIP readout circuit.

mission. The total time spent on the patch during a five-day flight is 103 hours, equivalent to an $86 \%$ observing efficiency, and the total sky coverage is $1133 \mathrm{deg}^{2}$. The observation program consists of at least two, five-day flights. The first flight will use the $150 \mathrm{GHz}$ configuration, while the second flight will use the 260 and $350 \mathrm{GHz}$ configuration. Instrument recovery and relaunch can be done quickly with Kiruna flights, so two flights in two successive years are possible.

\section{References}

[1] D. Baumann, et al., AIP Conference Series, 1141, 10, (2009).

[2] H. K. Eriksen, J. B. Jewell, C. Dickinson, A. J. Banday, K. M. Gorski, and C. R. Lawrence, ApJ, 676, $10,(2008)$.

[3] The Planck Collaboration. A\&A submitted, (2013). arXiv:1309.0382

[4] D. P. Finkbeiner, M. Davis, and D. J. Schlegel, ApJ, 524, 867, (1999).

[5] S. T. Staggs, N. C. Jarosik, S. S. Meyer, and D. T. Wilkinson, ApJ, L1, (1996).

[6] B. Reichborn-Kjennerud, et al., SPIE Conference Series, 7741, (2010).

[7] J. C. Bardin and S. Weinreb, IEEE MWCL, 19, 6, 407, (2008).

[8] A. Monfardini, et al., ApJS, 194, 24, (2011).

[9] L. J. Swenson, et al., SPIE Conference Series, 8452, (2012).

[10] J. Zmuidzinas, Annu. Rev. Condens. Matter Phys., 3, 169, (2012).

[11] C. McKenney, JOLT submitted, (2013).

[12] R. Duan, et al., SPIE Conference Series, 7741, (2010).

[13] http://casper.berkeley.edu 

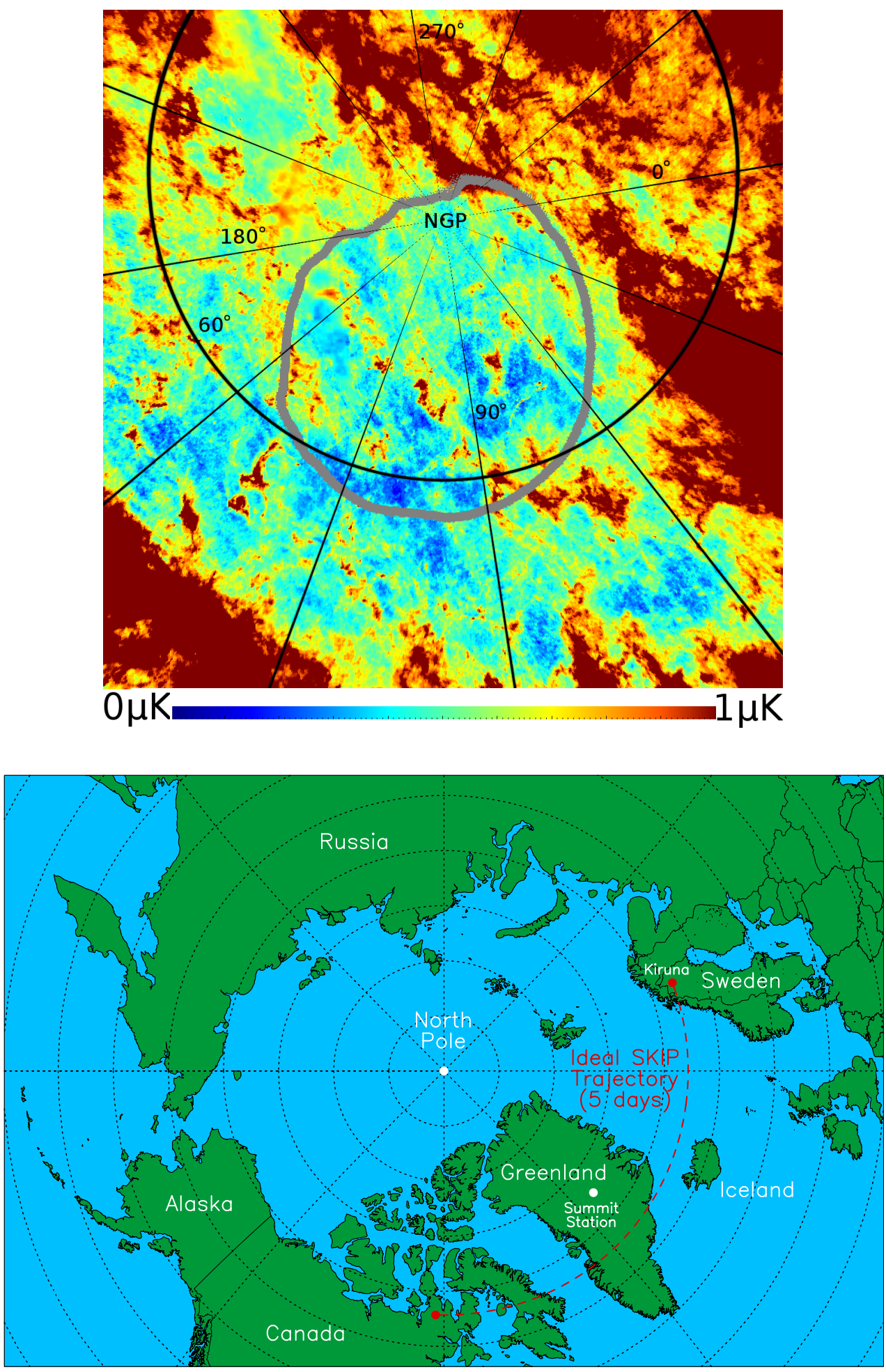

Figure 6: Top: Predicted polarized dust amplitude at $150 \mathrm{GHz}$ in the Northern celestial sky, assuming a 10\% dust polarization fraction [4]. The $1133 \mathrm{deg}^{2}$ SKIP target field is indicated by a gray boundary. Bottom: A map showing the anticipated SKIP trajectory. The balloon is launched in Kiruna, Sweden, and the instrument lands in Northern Canada five days later. 